\title{
Reintroduction of the PLB1 gene into Candida albicans restores virulence in vivo
}

\author{
Pranab K. Mukherjee, ${ }^{1} \dagger$ K. R. Seshan, ${ }^{2} \dagger$ S. D. Leidich, ${ }^{1}$ Jyotsna Chandra, ${ }^{1}$ \\ Garry T. Cole ${ }^{2}$ and Mahmoud A. Ghannoum ${ }^{1}$
}

Author for correspondence: Mahmoud A. Ghannoum. Tel: +1 216844 8580. Fax: +1 2168441076. e-mail: mag3@po.cwru.edu

\author{
1 Center for Medical \\ Mycology, University \\ Hospitals of Cleveland and \\ Department of \\ Dermatology, Case \\ Western Reserve \\ University, Cleveland, \\ OH 44106-5028, USA \\ 2 Department of \\ Microbiology and \\ Immunology, Medical \\ College of Ohio, Toledo, \\ OH 43614-5806, USA
}

\begin{abstract}
Phospholipases have been proposed to contribute to the virulence of Candida albicans. Recently, a candidal strain deleted for PLB1, the gene encoding the predominant phospholipase B (Plb1) secreted by C. albicans, was constructed and its virulence in an intravenous murine model of disseminated candidiasis was evaluated. In the present study, the PLB1 gene was reintroduced back into the plb1 null mutant to generate the revertant strain, which showed similar growth and morphology to its isogenic parent strain. Virulence of the revertant strain was found to be comparable to that of the parent strain in an intravenous murine model of disseminated candidiasis. To compare the abilities of the plb1 null mutant, the revertant and the isogenic parent strains to cross the gastrointestinal (GI) tract and cause systemic infection, an oral-intragastric infant mouse model of candidiasis was used. Histological examinations and analysis of c.f.u. of the pathogen in liver homogenates revealed that the parental and revertant strains were able to invade and traverse the GI mucosa to a significantly greater extent than the plb1 null mutant. Immunofluorescence and immunoelectron microscopic studies of infected host tissue using anti-Plb1 antibody showed that Plb1 is secreted during invasion of the gastric mucosa by the parental and revertant strains. In contrast, little or no labelling was observed in the null mutant strain. The results indicate that the Plb1 secreted by C. albicans enhances the ability of this organism to cross the GI tract and disseminate haematogenously. These studies provide unequivocal evidence supporting a role for Plb1 during the course of infection by $C$. albicans.
\end{abstract}

Keywords: phospholipase B, virulence factor, candidal transmigration, in vivo
localization of Plb1

\section{INTRODUCTION}

Infections caused by Candida albicans, the most common fungal pathogen of humans (Odds, 1994), have increased significantly in several patient populations, especially immunocompromised individuals (Odom, 1994; Jarvis, 1995; Viscoli et al., 1999). Candidaemia is the fourth leading cause of bloodstream infections in many intensive care units (Edmond et al., 1999; De Marie, 2000). Administration of antifungal agents, particularly amphotericin B and fluconazole, is the current mode of therapy used to treat candidal infections (Klepser et al., 1998; De Marie, 2000). However, renal

†These authors contributed equally to this work.

Abbreviations: GI, gastrointestinal; PAS, periodic acid-Schiff reagent. toxicity associated with amphotericin B and resistance to azole antifungals (including fluconazole) are inherent drawbacks in current antifungal therapies based on these drugs (Patterson \& Andriole, 1989). Even when the ideal antifungal treatment protocol has been established, mortality still persists at an unacceptably high range of 38-50\% (Wey et al., 1988, 1989; Pagano et al., 1999). In view of these obvious limitations of treatment with antifungal agents, it is evident that new approaches based on microbial virulence need to be developed for effective and less toxic antifungal drugs.

To develop such new therapies and treatments for candidiasis, it is essential to dissect the infectious process of C. albicans. Several virulence factors have been proposed for C. albicans, which may represent novel molecular targets for antifungal development. These 
factors include extracellular phospholipases and proteinases (Hube et al., 1998; Ghannoum, 2000; Mukherjee \& Ghannoum, 2001). Several bacterial and protozoan phospholipases have also been shown to contribute to the infectious processes of these pathogens (Ravdin et al., 1985; Saffer \& Schwartzman, 1991; Silverman et al., 1992; Schmiel \& Miller, 1999; Zhou et al., 2001). Like their bacterial and protozoan counterparts, the phospholipases of C. albicans also are considered important virulence determinants (Ibrahim et al., 1995), and could potentially facilitate increased penetration of fungal hyphal elements by directly damaging host cell membranes. To determine if phospholipases have a role in candidal virulence, we have previously cloned and disrupted PLB1, the gene encoding candidal phospholipase B (Plb1), and showed that virulence of the plb1 null mutant was significantly attenuated compared to that of its isogenic parental counterpart when tested in a murine model of haematogenously disseminated candidiasis (Leidich et al., 1998).

C. albicans is a member of the gastrointestinal (GI) microflora in normal individuals. In immunocompromised hosts, migration of this fungus across the GI tract represents one of the mechanisms by which disseminated candidiasis is established (Cole et al., 1989). An oral-intragastric infant mouse model of GI candidiasis has previously been established which is designed to mimic the process by which C. albicans traverses the human GI tract and haematogenously disseminates to other body organs (Cole et al., 1990, 1996). This model allows precise control of challenge dose and maintains the natural host barriers, e.g. gastric and intestinal secretions, peristalsis and mucin (Cole et al., 1996).

In our earlier investigations, we showed that the plb1 null mutant is attenuated in its virulence (Leidich et al., 1998). However, these studies did not involve a revertant strain containing the reintroduced PLB1 gene, due to the unavailability of such a strain at that time. To unequivocally prove the association of PLB1 with candidal pathogenesis, it was necessary to reintroduce the functional PLB1 gene back into the plb1 null mutant, and determine if the revertant has similar virulence to the parental strain. In this study, we report the successful construction of the PLB1 revertant strain and show that it has similar virulence to the wild-type strain in an intravenous murine model of haematogenously disseminated candidiasis. Additionally, using an oralintragastric infant mouse model of candidiasis, we provide evidence that Plb1p is secreted during $C$. albicans transmigration of the GI tract. Furthermore, deletion of the PLB1 gene results in significant reduction in the ability of the pathogen to traverse the stomach mucosa and disseminate haematogenously to the liver.

\section{METHODS}

Strains, plasmids and culture media. The C. albicans strains used in this study are listed in Table 1. Bacterial strain Escherichia coli DH5 $\alpha$ was used for propagation of all plasmids. The plasmid pPCR-Script Amp (SK+) (Stratagene) was used for subcloning experiments. Yeast strains were maintained at $4{ }^{\circ} \mathrm{C}$ on Sabouraud dextrose agar (SDA; $1 \%$ neopeptone, $2 \%$ glucose, $2 \%$ agar) (Difco Laboratories) and subcultured onto fresh SD medium every 1-2 weeks. Minimal defined media consisted of yeast nitrogen base (YNB) supplemented with $2 \%$ glucose. Media were supplemented with uridine $\left(50 \mu \mathrm{g} \mathrm{ml}^{-1}\right)$, and solidified with $2 \%$ agar as required. Unless noted otherwise, all cultures were incubated at $30^{\circ} \mathrm{C}$.

$\mathrm{Ura}^{-}$auxotrophs were selected on agar plates containing 5fluoroorotic acid and uridine $\left(50 \mu \mathrm{g} \mathrm{ml}^{-1}\right)$, as described previously (Boeke et al., 1984; Leidich et al., 1998). Dilutions of a suspension of PLB1 $\Delta^{2}$ cells were spread on these 5fluoroorotic acid plates, and scored after 3-4 d incubation. The loss of the URA3 gene in the resulting strains was confirmed by PCR and Southern blot analyses. A representative strain (PMY53) was randomly selected from among these $\mathrm{Ura}^{-}$derivatives for further experiments.

Reagents. Restriction endonucleases, T4 DNA ligase, DNA polymerase (Klenow fragment), calf intestinal alkaline phosphatase and Taq DNA polymerase were purchased from Boehringer Mannheim. Oligonucleotide DNA primers were synthesized by Bio-Synthesis. Glass beads $(25-600 \mu \mathrm{m})$, used for total chromosomal DNA extraction, and all other chemicals were obtained from Sigma. Guinea pig anti-Plb1 polyclonal antiserum was produced by Pocono Rabbit Farm \& Laboratories.

Standard procedures were followed for the propagation and selection of plasmids, the growth of their bacterial hosts, and for the subcloning of DNA fragments (Sambrook et al., 1989).

Construction of plasmids. To reintroduce the PLB1 gene, the integration plasmid pPMB3 was constructed as follows. Plasmid pMB-7, which contains the C. albicans URA3 gene flanked by $1 \cdot 1 \mathrm{~kb}$ direct repeats of the Salmonella typhimurium his G DNA, was kindly provided by Dr W. Fonzi (Georgetown University, Washington, DC, USA). A $2.9 \mathrm{~kb}$ fragment containing the URA3 gene was isolated by digesting pMB-7 with $\mathrm{BamHI}$ and cloned into the BamHI site of the plasmid pPCRScript Amp (Stratagene) to generate plasmid pPMB1. A SwaI/ApaI genomic fragment containing the intact PLB1 gene was ligated into the EcoRV site of plasmid pcDNA3.1 (Invitrogen) to form plasmid pGN. Digestion of pGN with EcoRI and XhoI led to the release of a $2 \cdot 2 \mathrm{~kb}$ PLB1 fragment, which was subcloned into the EcoRI/SalI sites of plasmid pPCR-Script Amp (Stratagene) to generate plasmid pPMB2. Next, plasmid pPMB2 was digested with $P s t \mathrm{I} / \mathrm{XhoI}$ to release the $2 \cdot 2 \mathrm{~kb}$ PLB1 fragment, which was then cloned into the Pst I/XhoI sites of plasmid pPMB1 to generate the integration plasmid pPMB3. The presence of URA3 and PLB1 genes in pPMB3 was confirmed by restriction enzyme analysis using URA3- or PLB1-specific restriction enzymes (data not shown). Integration plasmid pPMB3 was used in transformation of the plb1-disrupted strain of C. albicans.

Reintroduction of the C. albicans PLB1 gene. The integration plasmid (pPMB3, constructed as described above), containing the functional PLB1 and URA3 genes, was used to transform the null mutant strain in order to reintroduce these two genes. Plasmid pPMB3 was digested with $B g l \mathrm{II}$, which has a single recognition site in $P L B 1$ but not in the $U R A 3$ or vector regions (Fig. 1a). This linearized plasmid was then transformed into the PLB1 $\Delta^{2}$-derived $\mathrm{Ura}^{-}$strain (PMY53, generated by selection on 5-fluoroorotic acid as described above) using a lithium acetate-based transformation protocol (Rose et al., 1990). $\mathrm{Ura}^{+}$prototrophs were selected on minimal medium lacking uridine. Total chromosomal DNA was isolated (Rose et al., 
Table 1. C. albicans strains

\begin{tabular}{|c|c|c|}
\hline Strain & Genotype & Source/reference \\
\hline SC5314 & Wild-type & Gillum et al. (1984) \\
\hline CAI-4 & $\Delta u r a 3:: i m m 434 / \Delta u r a 3:: i m m 434$ & Fonzi \& Irwin (1993) \\
\hline $\operatorname{PLB} 1 \Delta^{2}$ & 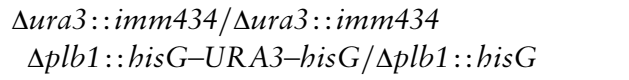 & Leidich et al. (1998) \\
\hline PMY53 & 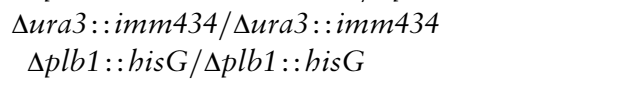 & This study \\
\hline PMY106 & $\begin{array}{l}\Delta \text { ura3::imm } 434 / \Delta u r a 3:: i m m 434 \\
\quad \Delta p l b 1:: \text { is } G / \Delta \text { plb1:: hisG-PLB1-URA3-hisG }\end{array}$ & This study \\
\hline
\end{tabular}

1990) from cultures produced by the growth of individual colonies (transformants). Insertion of the functional PLB1 gene into the null mutant as a result of spontaneous recombination was confirmed by Southern blot analyses.

PCR amplification of the PLB1-specific region. Total chromosomal DNA isolated from the respective strain was subjected to PCR with oligonucleotide primers identical to the $751 \mathrm{bp}$ region of the PLB1 gene. Oligonucleotide primers (BioSynthesis) used in the PCR reactions were: forward, $5^{\prime}$ ATGATTTTGCATCATTTG-3' ; reverse, 5'-AGTATCTGGAGCTCTACC-3'. PCR amplification reactions consisted of $100 \mu$ volumes containing $10 \mathrm{mM}$ Tris $/ \mathrm{HCl}(\mathrm{pH} \mathrm{8.4)}, 50 \mathrm{mM}$ $\mathrm{KCl}$, $1.5 \mathrm{mM} \mathrm{MgCl}_{2}$, dNTPs (1.0 mM each dNTP), oligonucleotide primers $(1 \mu \mathrm{M}$ each), template DNA (10-100 ng) and $2 \mathrm{U}$ Taq DNA polymerase. The PCR conditions were as follows: initial denaturation $\left(95^{\circ} \mathrm{C}, 2 \mathrm{~min}\right)$ followed by 33 cycles of denaturation $\left(94^{\circ} \mathrm{C}, 1 \mathrm{~min}\right)$, annealing $\left(50{ }^{\circ} \mathrm{C}, 1 \mathrm{~min}\right)$ and extension $\left(72^{\circ} \mathrm{C}, 3 \mathrm{~min}\right)$, ending with a $10 \mathrm{~min}$ extension step at $72{ }^{\circ} \mathrm{C}$. Reaction mixtures were analysed for the presence of a $751 \mathrm{bp}$ DNA fragment, representing the $5^{\prime}$ half of the PLB1 gene, on $1.2 \%$ agarose gels stained with ethidium bromide.

Southern blot analyses. Total chromosomal DNA was isolated from the respective C. albicans strains as described previously (Rose et al., 1990). The isolated DNA was digested with (i) $K p n \mathrm{I}$ and $S a c$ I or (ii) $K p n \mathrm{I}$ only [6 U ( $\mu \mathrm{g} \mathrm{DNA})^{-1}$ ], electrophoresed through $1.2 \%$ agarose and transferred to nylon membranes (Boehringer Mannheim) following standard protocols (Sambrook et al., 1989). The transferred DNA was cross-linked to the membrane using a UV Stratalinker 228 (Stratagene) delivering $120000 \mu \mathrm{J}$. The probes used were (a) a $3 \mathrm{~kb}$ HindIII fragment containing a portion of the $5^{\prime}$-terminus of the intact PLB1 gene, (b) a 736 bp BamHI fragment corresponding to the portion of PLB1 deleted during the disruption process (BamHI internal) (Fig. 1a) or (c) a $1.3 \mathrm{~kb}$ $X b a \mathrm{I}-P s t \mathrm{I}$ fragment of the PPMB1 plasmid which contains the $S$. typhimurium his $G$ region. Probes were labelled with digoxigenin (DIG)-dUTP using the DIG High Prime DNA Labelling and Detection Kit (Boehringer Mannheim) according to the manufacturer's instructions. Cross-linked membranes were prehybridized for $1 \mathrm{~h}$ at $65^{\circ} \mathrm{C}$, then hybridized overnight with DIG-labelled probe $\left(30 \mathrm{ng} \mathrm{ml}^{-1}\right)$ at $65^{\circ} \mathrm{C}$. After stringency washes, hybridizing DNA fragments were detected using the kit mentioned above, according to the manufacturer's instructions.

Western blot analysis and assays for Plb1 enzyme activities were performed with culture supernatants from the respective strains, as described previously (Leidich et al., 1998).

Haematogenous model of disseminated candidiasis. The protocol described earlier by our group was followed
(Ghannoum et al., 1995). Briefly, strains of C. albicans were grown in SD medium at $30^{\circ} \mathrm{C}$ until mid-exponential phase. Cells were harvested, washed, counted and resuspended at a

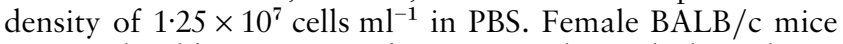
(7-8 weeks old) were injected intravenously via the lateral vein with $200 \mu \mathrm{l}\left(5 \times 10^{5}\right.$ blastospores $)$ of this fungal suspension. Cages were checked twice daily for dead or moribund mice. Mice were categorized as moribund if they displayed the following symptoms: lethargy, wasting and vertigo. Such mice were killed by $\mathrm{CO}_{2}$ asphyxiation. To determine tissue fungal burden, mice were injected with the respective $C$. albicans strain as described above. Mice in each group were killed $48 \mathrm{~h}$ post-infection and their kidneys were removed. The organs were weighed, homogenized separately in $5 \mathrm{ml}$ sterile PBS, and serial dilutions were plated on SDA plates supplemented with chloramphenicol $\left(50 \mu \mathrm{g} \mathrm{ml}^{-1}\right.$; Sigma). The plates were incubated at $30{ }^{\circ} \mathrm{C}$ for $24-48 \mathrm{~h}$, after which the number of c.f.u. was determined.

GI infection of mice (oral-intragastric model). Outbred mice [crl:CFW (SW) BR] obtained from Charles River Farms (Wilmington, MA, USA) were used to establish a breeding colony and the offspring of these animals were used in all experiments. Infant mice, $6 \mathrm{~d}$ old $(3-4 \mathrm{~g})$, were separated from their mother $4 \mathrm{~h}$ prior to challenge. Candidal cells grown on SDA slants at $30^{\circ} \mathrm{C}$ were harvested, washed with saline, and the challenge dose was standardized by haemocytometer counts. The inoculum size was confirmed by dilution plating. Infant mice were inoculated with $2 \times 10^{8}$ blastospores in a total volume of $0.05 \mathrm{ml}$ by the oral-intragastric route using a 24-gauge animal feeding needle (Popper \& Sons) as described previously (Cole et al., 1990). Following inoculation, the pups were kept at $35^{\circ} \mathrm{C}$ for $1 \mathrm{~h}$ before being returned to their mother. We determined that C. albicans delivered by this route was not recovered from the lungs or blood of the pleural cavity when these tissues were cultured within 10 min postchallenge, thus making it unlikely that spread to body organs resulted from faulty inoculation technique or aspiration.

Faecal pellet assay and separation of test groups. Mice infected by oral-intragastric inoculation with $C$. albicans were selected for further study on the basis of the presence of yeast in their faecal pellets at $9 \mathrm{~d}$ post-challenge (Cole et al., 1989). Animals were marked for identification using picric acid at the time of the assay. Fresh faecal pellets were immediately homogenized in $1.0 \mathrm{ml}$ chilled, sterile saline and $0.1 \mathrm{ml}$ of the homogenate was plated on SDA (Difco) supplemented with $50 \mu \mathrm{g}$ chloramphenicol $\mathrm{ml}^{-1}$. The plates were incubated at $37^{\circ} \mathrm{C}$ for $48 \mathrm{~h}$. Mice identified as culture positive for C. albicans were immunocompromised as described below and used to compare the systemic spread of the parental (SC5314), plb1 null mutant $\left(\mathrm{PLB} 1 \Delta^{2}\right)$ and revertant (PMY106) strains from the site of colonization in the GI tract. 
(a)
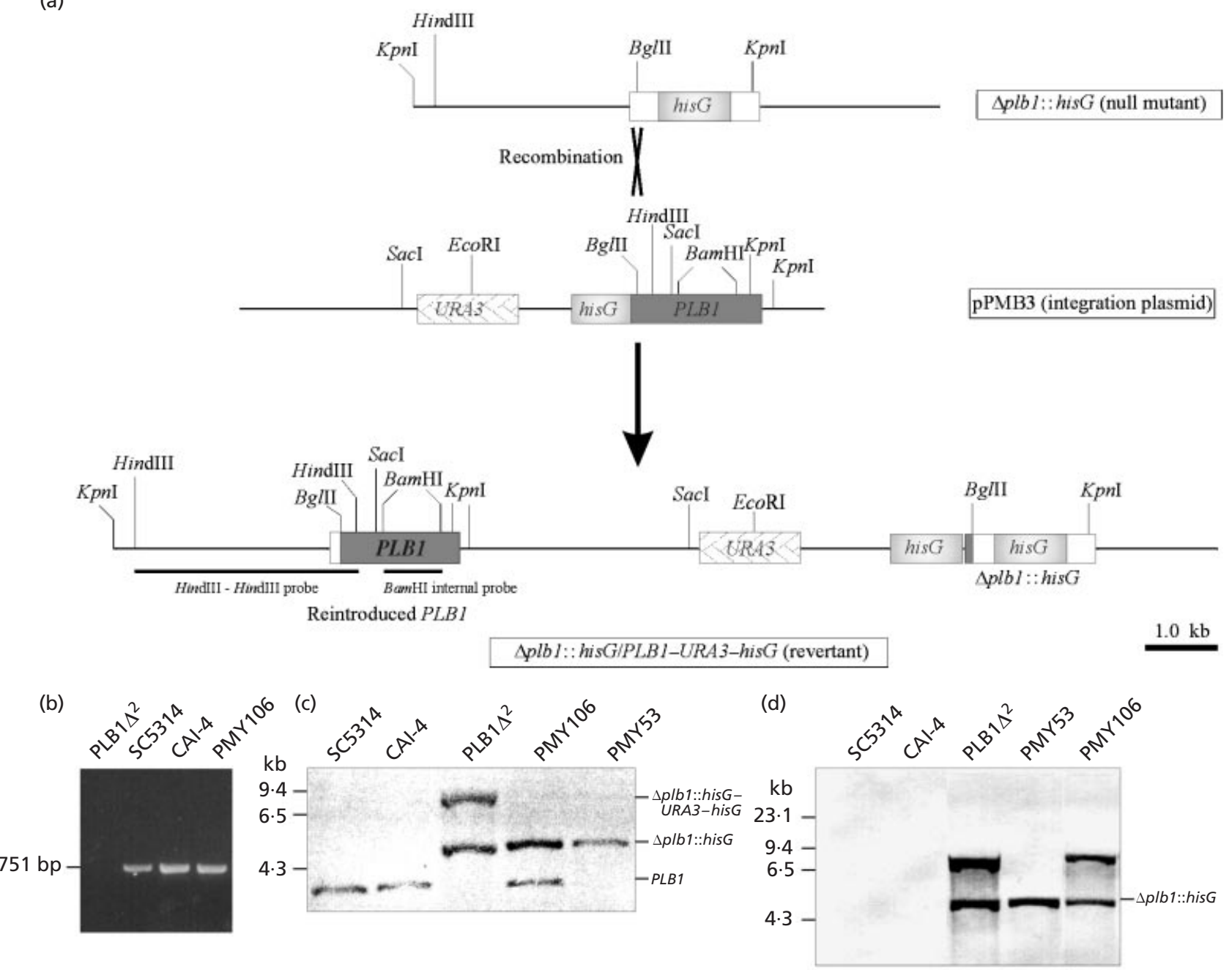

Fig. 1. Reintroduction of the PLB1 gene. (a) Scheme followed to construct the revertant strain. The null mutant was transformed with the $B g / l l-d i g e s t e d$ integration plasmid pPMB3. Spontaneous recombination at the unique $B g / l l$ site of $\Delta p / b 1::$ hisG in the null mutant resulted in the formation of the revertant. (b) PCR amplification of the targeted $751 \mathrm{bp}$ PLB1 region (arrow) was observed in chromosomal DNA isolated from the revertant (PMY106) and parental (SC5314 and PMY53) strains, but not in the mutant $\left(\right.$ PLB1 $\left.\Delta^{2}\right)$. (c) Southern blot analysis of chromosomal DNA isolated from the respective strains (each digested with $\mathrm{Kpnl}$ and Sacl) using the PLB1-specific Hindlll probe. A $3.6 \mathrm{~kb}$ fragment corresponding to the intact PLB1 was seen in the revertant as well as the parental strains but not in the mutant strain, which contained a $4.7 \mathrm{~kb}$ fragment corresponding to the disrupted $\Delta p / b 1::$ his . The $7.6 \mathrm{~kb}$ fragment observed in PLB1 $\Delta^{2}$ corresponds to the $\Delta p / b 1::$ hisG-URA3-hisG disrupted copy of the gene. (d) Parental, revertant and mutant strains were probed for the presence of the bacterial hisG sequence by hybridizing Kpnl-digested chromosomal DNA with the $1.3 \mathrm{~kb}$ Pstl-Xbal hisG-specific probe (derived from the plasmid pPMB1). Strains PLB1 ${ }^{2}$, PMY53 and PMY106 showed the presence of the hisG region, while as expected SC5314 and CAI-4 did not hybridize to the probe. The 7.6 and $4.7 \mathrm{~kb}$ fragments (corresponding to the $\Delta p / b 1:: h i s G$ and $\Delta p / b 1:: h i s G-U R A 3-h i s G$ regions, respectively) were expected in PLB1 $\Delta^{2}$, while the single $4.7 \mathrm{~kb} \Delta$ plb1::hisG fragment was detected in PMY53. Strain PMY106 contained the $4.7 \mathrm{~kb}$ $\Delta p / b 1:: h i s G$ and the $8 \mathrm{~kb} U R A 3-h i s G-\Delta p / b 1:: h i s G$ fragments.

Immunosuppression. An immunocompromising treatment was initiated on day 11 after oral-intragastric challenge as previously reported (Cole et al., 1989). Mice selected on the basis of positive faecal pellets received cyclophosphamide (Adria Laboratories) by the intraperitoneal route at doses of $0 \cdot 2 \mathrm{mg}(\mathrm{g} \text { body } \mathrm{wt})^{-1}$ and $0 \cdot 1 \mathrm{mg}(\mathrm{g} \text { body t})^{-1}$, on days 11 and 14 post-challenge, respectively. In addition, $1.25 \mathrm{mg}$ cortisone acetate (Merck, Sharp \& Dohme) was administered by the intraperitoneal route on days 11 and 14 post-challenge. This combined drug treatment was chosen on the basis of its severe immunocompromising effects, which lead to systemic spread of C. albicans from sites of colonization in the GI tract (Guentzel \& Herrera, 1982; Cole et al., 1991a). Mice were killed at day 20 post-challenge by $\mathrm{CO}_{2}$ asphyxiation. Stomachs and livers were aseptically removed and prepared for microscopic analyses or quantification of fungal c.f.u. as described previously (Pope et al., 1979). For enumeration of candidal c.f.u., the stomach and liver of each infected animal were removed under aseptic conditions, visually inspected for gross candidal foci, and homogenized separately in $5.0 \mathrm{ml}$ sterile saline (Travenol Laboratories) using a tissue grinder equipped with a Teflon pestle (Cole et al., 1989). The c.f.u. of C. 
albicans in each organ were determined by plating serial dilutions of the respective homogenate on SDA supplemented with chloramphenicol $\left(50 \mu \mathrm{g} \mathrm{ml}^{-1}\right)$ as above. Plates were incubated at $37^{\circ} \mathrm{C}$ for $48 \mathrm{~h}$ and then colonies were counted.

Light microscopy. The stomachs of five animals from each group (parental, plb1 null mutant and revertant strain infected) were used for light microscopic histological and immunofluorescence labelling. In addition, stomachs of five more animals from each group were used for electron microscopic and immunogold labelling studies. Following removal, stomachs were immediately placed in chilled saline and dissected open to expose the cardial-atrial fold (Cole et al., 1990). Tissues were fixed in $4 \%$ paraformaldehyde $(\mathrm{v} / \mathrm{v}$ in PBS; $0 \cdot 1 \mathrm{M}, \mathrm{pH} 7 \cdot 4)$ for $12 \mathrm{~h}$ at $4{ }^{\circ} \mathrm{C}$. Tissues were then washed with buffer, dehydrated in ethanol, and embedded in paraffin wax. Sections were stained with periodic acid-Schiff reagent (PAS) for demonstration of fungal elements (Luna, 1968).

Transmission electron microscopy. C. albicans-infected stomachs were chemically fixed for $12 \mathrm{~h}$ at $4{ }^{\circ} \mathrm{C}$ in glutaraldehyde $(3 \%, \mathrm{v} / \mathrm{v})$ and paraformaldehyde $(2 \%, \mathrm{v} / \mathrm{v})$, each prepared separately in cacodylate buffer $(0 \cdot 1 \mathrm{M}, \mathrm{pH} 7 \cdot 4)$ and mixed just before use. The tissues were rinsed five times in buffer, post-fixed in $2 \%$ osmium tetroxide $(2 \mathrm{~h})$ prepared in the same buffer, dehydrated and embedded in Spurr's low viscosity resin as described previously (Seshan \& Cole, 1994). Thick sections (approx. $1 \mu \mathrm{m}$ ) were stained with azure 11methylene blue for light microscopy as reported by Cole et al. (1989). Thin sections were mounted on copper grids, stained with uranyl acetate and lead citrate and examined with a Phillips CM-10 transmission electron microscope. Osmium tetroxide post-fixation was omitted for tissues intended for immunofluorescence and immunogold labelling described below.

Production of antibodies. Guinea pig anti-Plb1 polyclonal antibodies were produced commercially (Pocono Rabbit Farm \& Laboratory). A standard protocol was followed for the antibody production. Briefly, on day 0 a preimmune (baseline) bleed was performed. This preimmune serum was stored at $-80{ }^{\circ} \mathrm{C}$ until assayed. On the same day, $50 \mu \mathrm{g}$ purified Plb1 was injected intramuscularly in Freund's complete adjuvant. On day 14, 10-20 $\mu \mathrm{g}$ Plb1 was injected intramuscularly in Freund's incomplete adjuvant. A test bleed was performed on day 42 post-immunization and anti-Plb1 antibody levels were titred. Guinea pigs were boosted every 4 weeks with $10 \mu \mathrm{g}$ Plb1 antigen until peak antibody titres were achieved. Antiserum samples were stored frozen $\left(-80^{\circ} \mathrm{C}\right)$ until assay.

Immunofluorescence microscopy. Immunofluorescence detection of Plb1 was performed on chemically fixed, resinembedded stomach tissue harvested from mice infected with the parent, plb1 null mutant or revertant strains. Ten thick $(1.0 \mu \mathrm{m})$ sections of stomach tissues of each strain cut with an ultramicrotome (MT-5000; Dupont) were mounted on a specially prepared gelatin-coated glass slide for immunolabelling. Sections were blocked with $1 \%$ bovine serum albumin in PBS $(0 \cdot 15 \mathrm{M}, \mathrm{pH} 7 \cdot 4)$ for $10 \mathrm{~min}$, reacted with primary antibody (guinea pig anti-Plb1) diluted 1:500 in PBS for $1 \mathrm{~h}$ and incubated with goat anti-guinea pig IgG-FITC secondary antibody (Sigma; diluted 1:30 in PBS) for $1 \mathrm{~h}$. The sections were washed and examined with a Zeiss microscope equipped with an FITC filter set. Control sections were reacted either with FITC alone, or with normal guinea pig preimmune serum followed by secondary antibody-FITC conjugate. These experiments were repeated five times.

Cryofixation and freeze-substitution. To better preserve the association between the soluble Plb1p antigen and the fungal cells, the infected tissue was subjected to cryofixation and freeze-substitution (Cole et al., 1991b). In brief, blocks of dissected stomach tissue (approx. $3 \mathrm{~mm}^{2}$ ) from the region of the cardial-atrium fold were dropped onto a polished copper block prechilled with liquid nitrogen. The frozen tissue was then immediately transferred to a vial containing the substitution fluid (anhydrous acetone plus $0.05 \%$ uranyl acetate), which was maintained at $-80^{\circ} \mathrm{C}$. The tissue was then freezesubstituted at $-80^{\circ} \mathrm{C}$ for $48 \mathrm{~h},-40^{\circ} \mathrm{C}$ for $24 \mathrm{~h},-20^{\circ} \mathrm{C}$ for $24 \mathrm{~h}, 4^{\circ} \mathrm{C}$ for $4 \mathrm{~h}$, and then gradually brought to room temperature. After two rinses with absolute acetone, the tissues were embedded in Spurr's resin (Spurr, 1969).

Immunogold labelling for localization of Plb1. Stomach tissue was prepared for immunoelectron microscopy as previously described (Kruse \& Cole, 1992). Briefly, three to four thin sections of cryofixed, infected stomach tissue were mounted on a Formvar-coated nickel grid. A total of 15 grids containing about 45-60 thin sections from each group were used for immunological labelling. The grids were floated on $10 \%$ ovalbumin in $20 \mathrm{mM}$ Tris $/ \mathrm{HCl}$ (pH 7.4) for $10 \mathrm{~min}$. The grids were then transferred to droplets of the guinea pig anti-Plb1 primary antibody (diluted 1:500) prepared in $1 \%$ ovalbumin in $\mathrm{Tris} / \mathrm{HCl}$ buffer. Control sections were incubated in preimmune guinea pig serum diluted as above. Sections were incubated for $48 \mathrm{~h}$ at $4{ }^{\circ} \mathrm{C}$. The grids were rinsed with buffer and incubated on droplets of goat anti-guinea pig secondary antibody conjugated with colloidal gold ( $20 \mathrm{~nm}$ diameter). The secondary antibody was prepared in Tris/ $\mathrm{HCl}$ buffer $(\mathrm{pH}$ $8 \cdot 2$ ) and diluted $1: 20$ in $1 \%$ ovalbumin. The grids were finally washed in buffer followed by distilled water, and then stained with uranyl acetate and lead citrate. The specimens were examined with a transmission electron microscope as described above.

Statistical analysis. The numbers of c.f.u. per organ were expressed on a log scale. Because these values did not fall into normal distribution, the Mann-Whitney U test was used to compare medians. Statistical comparisons were performed using the StatView version 4.5 software package for Windows 95, or SPSS version 9.0 software package for Windows.

\section{RESULTS}

The strategy followed for the reintroduction of the PLB1 gene into the mutant PLB1 $\Delta^{2}$ strain is shown in Fig. $1(\mathrm{a})$. Spontaneous recombination between the chromosomal DNA and the BglII-linearized integration plasmid (pPMB3) is expected to result in the formation of the revertant strain PMY106, which contains both the PLB1 and URA3 genes.

\section{Reintroduction of the functional PLB1 gene}

Strain PMY53 (the $\mathrm{Ura}^{-}$derivative of the plb1 null mutant) was selected as the recipient for transformation with the BglII-digested integration plasmid pPMB3 containing a functional PLB1 gene. Growth and morphology of the resulting transformants were compared and found to be similar (data not shown); one of these transformants, PMY106, was selected as the representative revertant strain and further analysed. PCR analysis was performed to determine the correct insertion of the functional PLB1 gene into PMY53. Total chromosomal DNA was isolated from PMY106 and subjected to PCR using a PLB1-specific $751 \mathrm{bp}$ region as template. Fig. 1(b) shows that the revertant (PMY106) and 


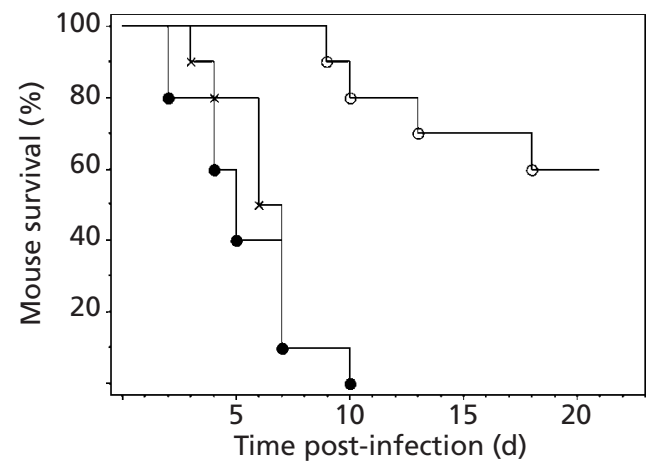

Fig. 2. Survival of mice following experimentally induced candidiasis (haematogenously disseminated model). The virulence of the revertant ( $X$, PMY106) strain was compared with the parental $(0, \mathrm{SC} 5314)$ and plb1 null mutant $(O$, PLB1 $\Delta^{2}$ ) strains in an intravenous murine model of haematogenously disseminated candidiasis. BALB/c mice (10 per group) were infected via the lateral tail vein with $5 \times 10^{5}$ blastospores of the respective $C$. albicans strain. Cages were checked twice daily for dead or moribund mice. Survival was monitored for $21 \mathrm{~d}$.

parental (SC5314 and CAI-4) strains contained the functional PLB1 allele amplified as a $751 \mathrm{bp}$ fragment, while no signal corresponding to the amplified functional PLB1 could be detected in the null mutant.

To confirm the reintroduction of the PLB1 gene into the mutant strain, total chromosomal DNA isolated from the transformant PMY106 was analysed by Southern blotting using a C. albicans PLB1-specific, as well as a bacterial his G-specific probe. Fig. 1(c) shows that the $3.6 \mathrm{~kb}$ band corresponding to the functional PLB1 gene present in the parent SC5314 and CAI-4 strains is also detected in PMY106, thus proving the introduction of functional PLB1 in the transformant. To make sure that PMY106 was not a contaminant strain, total chromosomal DNA from the revertant strain was digested with $K p n \mathrm{I}$, and hybridized with a his G-specific probe. An $8 \mathrm{~kb}$ band representing the URA3-hisG-
$\Delta p l b 1:$ : his $G$ fragment and a $4.7 \mathrm{~kb}$ band representing the $\Delta p l b 1:$ : his $G$ fragment were detected (Fig. 1d). The parent strains (SC5314 and CAI-4) did not show any detectable fragment, which was expected since the probe used (his $G$ ) represents a bacterial sequence. These results clearly show that the PLB1 gene was successfully reintroduced into the mutant strain.

Western blot analysis of the culture supernatant from the revertant, mutant and parental strains was performed to detect the Plb1 protein secreted by these strains. Plb1p was detected in both the parental and revertant strains, but not in the mutant strain (data not shown). Additionally, the relative levels of Plb1 enzyme activity in culture supernatant from the parental, revertant and mutant strains were $100 \%, 98 \%$ and $1 \%$, respectively, as determined by the colorimetric free fatty acid assay procedure. This indicated that the enzyme activity in the revertant strain was similar to that of the parental strain.

\section{Reintroduction of PLB1 restores candidal virulence (haematogenously disseminated model)}

A murine model of haematogenously disseminated candidiasis was employed to evaluate the effect of PLB1 reintroduction on virulence. All mice injected with either the wild-type strain SC5314 or the revertant PMY106 strain succumbed to candidal infection within $10 \mathrm{~d}$ (Fig. 2). In contrast, $80 \%$ of the mice injected with PLB1 $\Delta^{2}$ were alive at day 10 . The mean survival time $( \pm \mathrm{SD})$ for mice infected with SC5314 was $5 \cdot 30 \pm 0 \cdot 790 \mathrm{~d}$, while that for mice infected with the revertant strain was $6.30 \pm 0.59 \mathrm{~d}$. In contrast, the mean survival time for mice infected with the plb1-disrupted mutant was $15 \cdot 80 \pm 1 \cdot 27 \mathrm{~d}$. Statistical comparisons of the survival curves by Logrank (Mantel-Cox) analysis revealed that survival of mice infected with strain SC5314 did not differ significantly from that of mice infected with PMY106 $(P=0 \cdot 48)$. In contrast, mice infected with the PLB1 $1 \Delta^{2}$ mutant strain survived significantly longer than mice infected with either SC5314 or PMY106 $(P<$

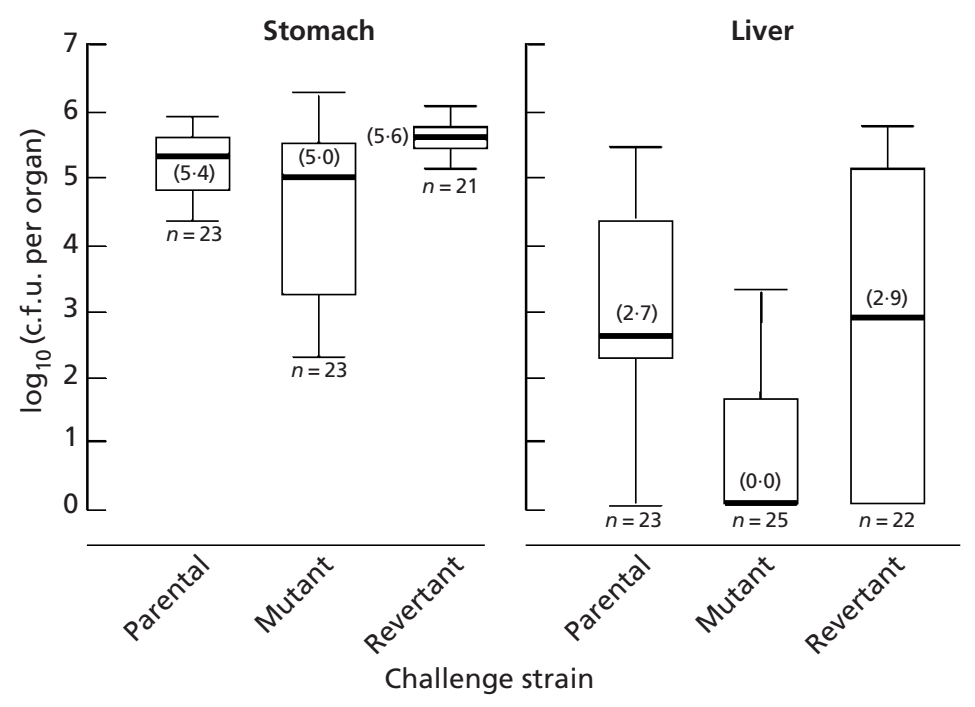

Fig. 3. Box plot representation of the number of c.f.u. detected in the stomach and liver of immunocompromised mice (oral-intragastric model). Mice were challenged by the oral-intragastric route with the parental, revertant or Plb1deficient strains of $C$. albicans. The boxes indicate the 25th and 75th percentiles, and the bars show the 5th and 95th percentiles. The line within the box indicates the median. 

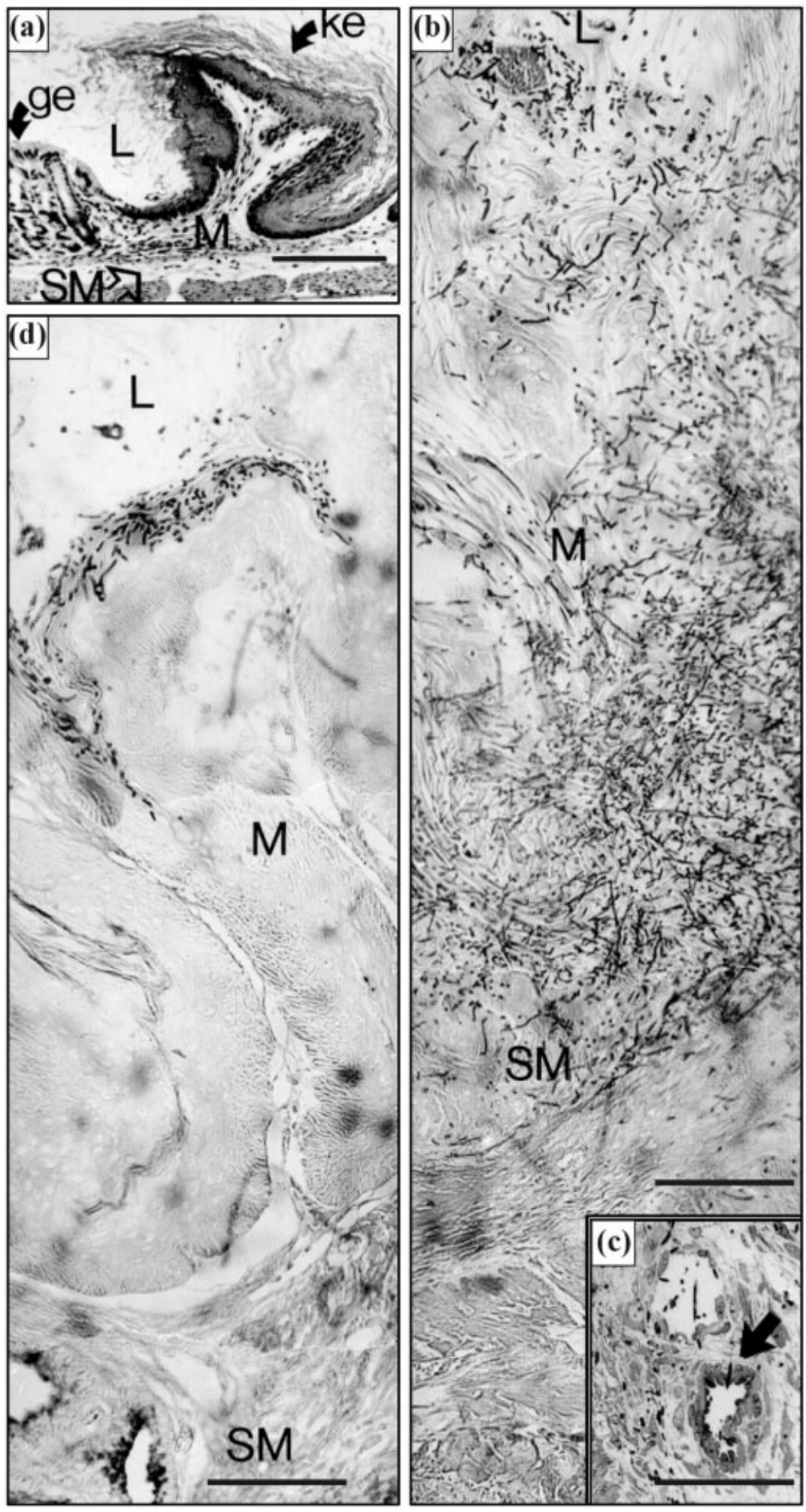

Fig. 4. Histological comparison of the invasiveness of the parental and Plb1deficient strains of C. albicans. (a) PASstained section of the stomach of a noninfected, 4-week-old mouse in the region of the cardial-atrium fold (ge, glandular epithelium; ke, keratinized epithelium; L, lumen). (b) Section of the cardial-atrium fold of a mouse infected with the parental strain showing the extensive invasion of hyphal and yeast elements within the mucosal (M) and submucosal (SM) layers. (c) Blood vessel in the submucosal region of the stomach shown in (b) which reveals a hyphal element (arrow) that has crossed the endothelial layer. (d) Same region of the murine stomach as (b) but infected with the Plb1deficient $C$. albicans strain showing superficial and less abundant hyphal elements. Bars, $200 \mu \mathrm{m}(\mathrm{a})$ and $100 \mu \mathrm{m}$ (b, c, d).
0.0001 for both comparisons). Candidal c.f.u. were recovered from the kidneys of mice challenged with either the parental (SC5314) or revertant (PMY106) strain. No significant difference was found $(P=0.54)$ between c.f.u. count for mice challenged with the revertant strain $\left[1 \cdot 3 \pm 0 \cdot 12 \times 10^{6} \quad\right.$ c.f.u. $\left.(\mathrm{g} \text { tissue })^{-1}\right]$ and those challenged with the parental strain $\left.\left[1 \cdot 2 \pm 0 \cdot 28 \times 10^{6} \text { c.f.u. (g tissue) }\right)^{-1}\right]$. Strain PMY106 recovered from the kidneys of infected mice was incubated in serum for $2 \mathrm{~h}$, and no difference was found in its ability to form germ tubes, as compared to SC5314 (data not shown). Revertant and wild-type strains 

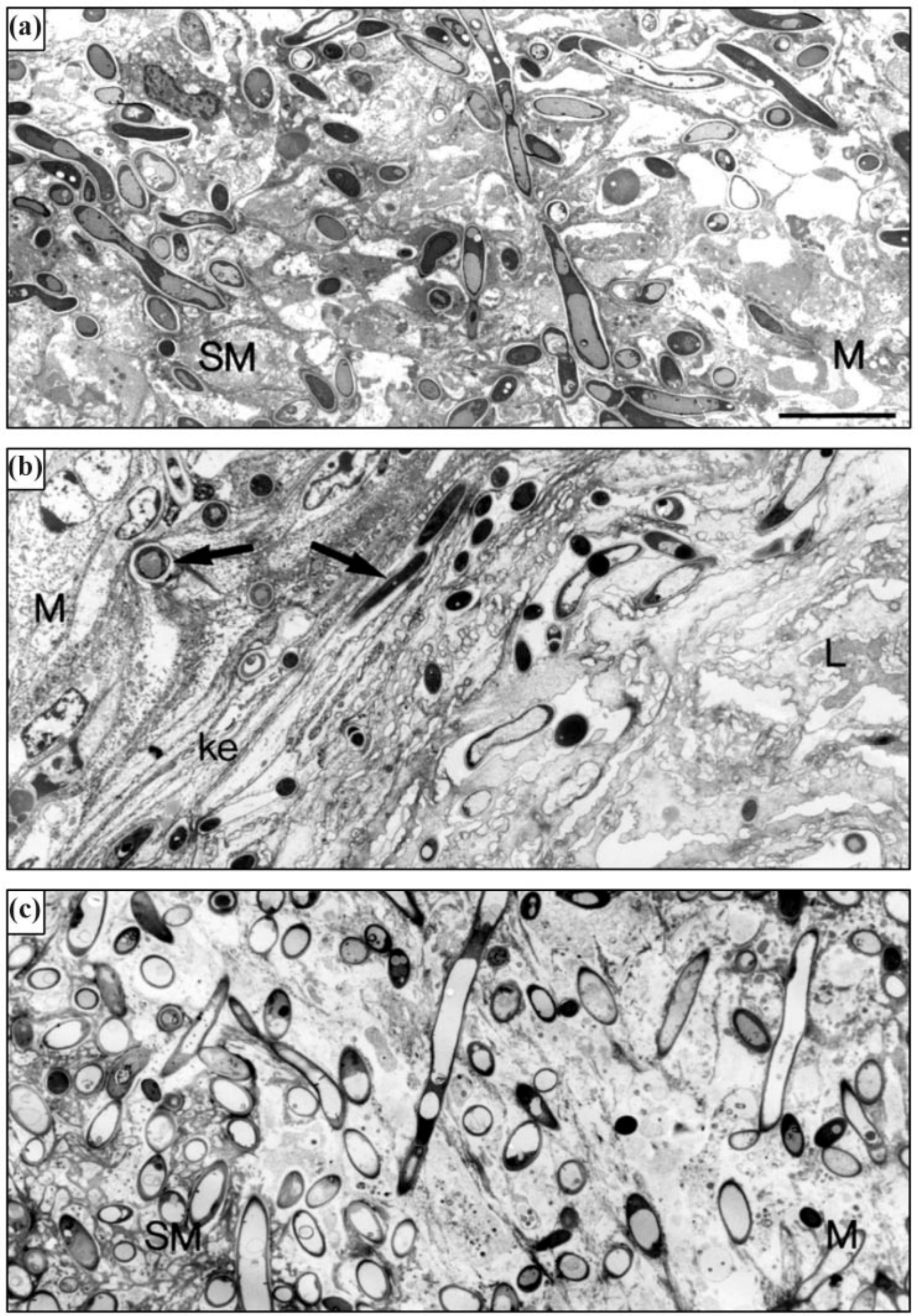

Fig. 5. For legend see facing page. 
passed through mice were further analysed by Southern hybridization, which confirmed that passage through animals had no effect on the genetic composition of these strains (data not shown). These results show that the reintroduction of the PLB1 gene restores the virulence properties of C. albicans in the haematogenously disseminated model of candidiasis.

\section{Fungal colonization of stomach and liver (oral-intragastric model)}

The numbers of C. albicans c.f.u. recovered from the stomach and liver of mice challenged with the parental, plb1 null mutant or revertant strains were compared. c.f.u. counts of the three strains in the stomachs of infected mice were not significantly different, as revealed by the box plots in Fig. 3. However, the c.f.u. in the liver were significantly greater in mice challenged with the parental $(P=0.0001)$ or revertant $(P=0.006)$ strains compared to mice challenged with the plb1 null mutant. In addition, the number of mice that developed systemic candidiasis infection differed markedly following challenge with the three strains. Ninety per cent and $70 \%$ of mice challenged with the parental or revertant strains exhibited liver colonization, respectively. In contrast, only $45 \%$ of mice infected with the plb1 null mutant exhibited liver involvement, and low numbers of c.f.u. were recovered from organ homogenates from these mice.

\section{Candidal invasion of gastric mucosa}

The histopathological appearance and localization of $C$. albicans in the immunocompromised mice challenged with the parental, revertant and mutant strains showed marked differences. The focus of these studies was the cardial-atrium fold of the stomach (Fig. 4a), where C. albicans is known to concentrate in mice after oralintragastric challenge (Cole et al., 1990). In PAS-stained sections of the stomach of mice infected with the parental strain, both hyphal and yeast elements occurred in large numbers in the gastric mucosa and submucosa (Fig. 4b). Hyphal elements, which had apparently penetrated the endothelial layer of blood vessels, were frequently found (Fig. 4c). Similar observations were recorded for mice challenged with the revertant strain (data not shown). In contrast, hyphae of the plb1 null mutant appeared to be restricted to the lumen and exposed epithelial surface of the gastric mucosa (Fig. $4 \mathrm{~d})$, and were very rarely observed deeper in the submucosa.

Thin sections of the stomach of mice in the region of the cardial-atrium fold which were infected with the parental strain of $C$. albicans consistently revealed invasive hyphae and yeast cells associated with necrotic host tissue (Fig. 5a). In sharp contrast, most hyphae of the plb1 null mutant were associated with the keratinized layer in this same region of the murine stomach, and apparently were unable to penetrate into the gastric submucosa tissue layers (Fig. 5b). Thin sections of the stomach of mice infected with the revertant strain (Fig. $5 \mathrm{c})$ showed essentially the same morphological features as the gastric tissue of mice infected with the parental strain.

\section{Phospholipase B expression in vivo}

Expression of Plb1p during the course of infection in the stomach was investigated using indirect immunofluorescence as well as immunoelectron microscopy. Guinea pig antiserum raised against the purified Plb1 protein reacted with hyphal and yeast cell walls of the parental strain in vivo, as revealed by immunofluorescence (Fig. 6a) as well as by immunoelectron microscopy (Fig. 6b). The plb1 null mutant, on the other hand, showed little to no label (Fig. 6c, d). The intensity of label in the walls of hyphae and yeast cells of the revertant strain was comparable to that of the parental strain (Fig. 6e, f). Control sections incubated with preimmune serum followed by secondary antibody did not produce any detectable fluorescence (Fig. $6 \mathrm{~g}$ ) or immunogold label (Fig. 6h).

\section{DISCUSSION}

The results of this study provide further evidence in support of Plb1 as a virulence determinant for $C$. albicans. We constructed a PLB1 revertant strain by reintroducing the PLB1 gene into the null mutant $\left(\operatorname{PLB} 1 \Delta^{2}\right)$, and showed that the revertant strain had similar growth, morphology and Plb1p secretion properties to the parental SC5314 strain. Successful construction of the revertant strain provided us with a set of isogenic strains differing only at the PLB1 locus. Using this set of isogenic strains in a haematogenously disseminated murine model of candidiasis, we showed that the revertant strain had similar virulence to the parental strain, but significantly greater virulence than the null mutant. Additionally, employing an oralintragastric infant mouse model of disseminated candidiasis, we showed that the revertant strain is similar to SC5314 in its efficiency of invading the GI tract and colonizing the host liver. In contrast, the plb1 null mutant was much less efficient at invading and colonizing host tissues, compared to the SC5314 or revertant strains. These findings from two different animal models of disseminated candidiasis are in agreement with our previous studies in which disruption of the PLB1 gene led to the attenuation of candidal virulence in a murine model of candidiasis (Leidich et

Fig. 5. Electron microscopic images of the gastric mucosa of immunocompromised mice infected with $C$. albicans. (a) Thin section showing numerous hyphal elements and yeast cells in the gastric mucosa (M) and submucosa (SM) of a mouse infected with the parental strain of $C$. albicans. (b) Hyphal elements of the Plb1p-deficient mutant present in the gastric lumen (L) and associated with the keratinized epithelium (ke; arrows) of the stomach at $20 \mathrm{~d}$ post-infection. (c) Hyphae and yeast cells of the revertant strain present in the gastric mucosa (M) and submucosa (SM) at $20 \mathrm{~d}$ post-challenge. Bar, $10 \cdot 0 \mu \mathrm{m}$. 

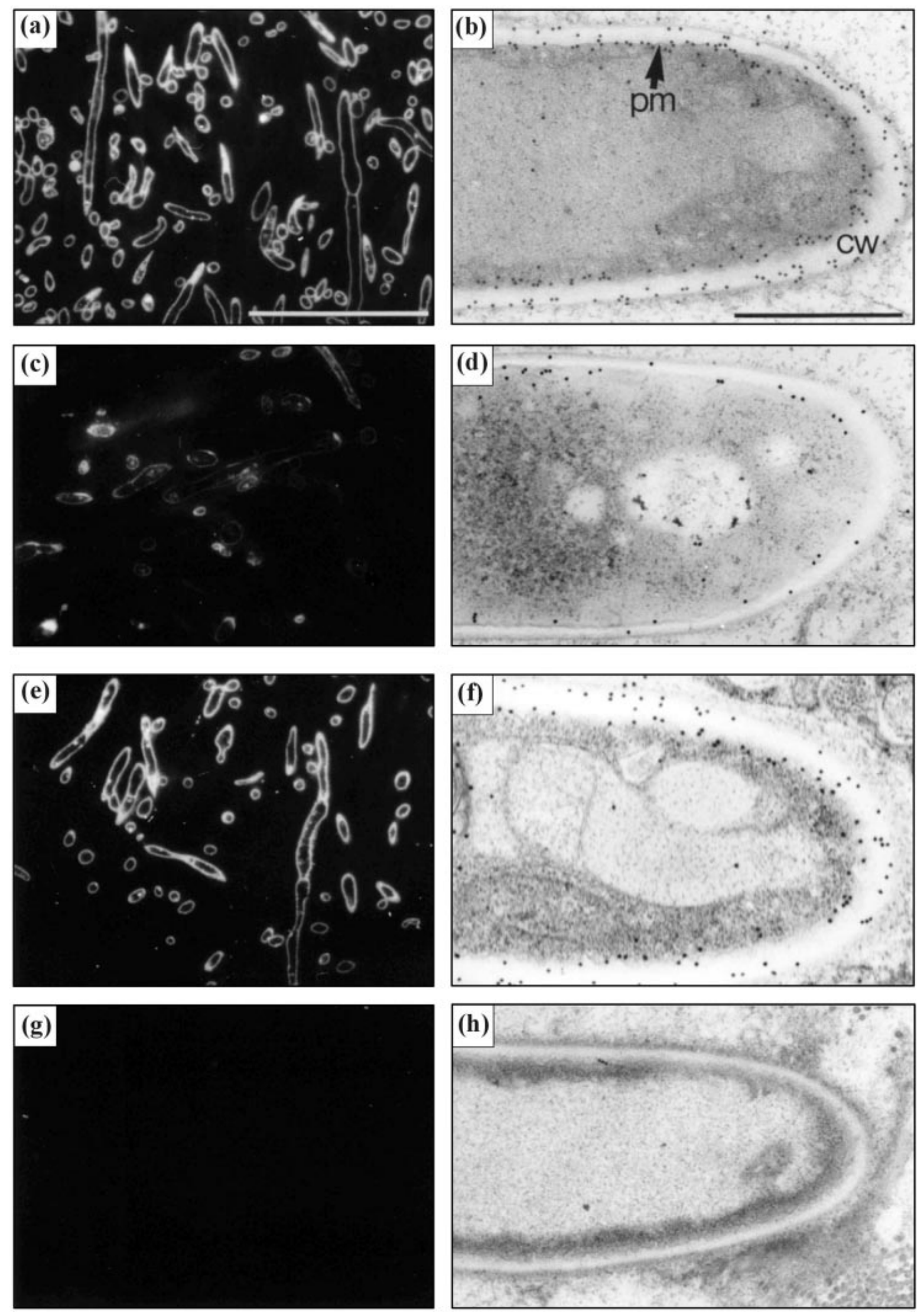

Fig. 6. For legend see facing page. 
al., 1998). The finding that the revertant strain can restore virulence in C. albicans to near wild-type levels has also been observed in other studies. Thus, the pathogenicity of C. albicans was restored to a near wildtype degree upon reintroduction of the VPS34 (vacuolar protein sorting) gene into the corresponding null mutant (Bruckmann et al., 2000). Additionally, a Klaac null mutant of the fungus Kluyveromyces lactis was functionally complemented by the introduction of a single copy of the Saccharomyces cerevisiae AAC1 (ADP/ATP carrier) gene (Viola et al., 1999). In another study, the cgt1/CGT1 (C. albicans mRNA 5'guanylyltransferase) heterozygote was found to be equally virulent for mice and guinea pigs when tested in an intravenous infection model of disseminated candidiasis (De Backer et al., 2000). Thus, at least some genes in C. albicans do not appear to be dose-dependent in expressing their phenotypic characteristics.

The mechanism/s by which Plb1p contributes to virulence is not well understood, but probably involves direct damage or degradation of host cell membranes. Such host cell injury would be expected to facilitate candidal penetration. In this context, Klotz et al. (1983) used an in vitro model to depict the earliest events of metastatic C. albicans infection and showed that the pathogen first adheres to, and then penetrates the endothelium. During transmigration, endothelial cell continuity was found to be disrupted by the yeasts. As destruction of the endothelium progressed, the fungus penetrated deeper into the substance of the vascular tissue. These authors attributed the dissolution of a portion of the endothelial cells to phospholipase activity. In another study, Pugh \& Cawson (1977) reported that phospholipase activity is generally localized at the tips of developing hyphae, i.e. in the direction of candidal penetration. Consistent with these findings, our immunolabelling studies showed that Plb1 localized mostly to the hyphal tips. Although the cell wall of plb1 null mutant cells reacted slightly with the immunolabel, the intensity of the signal was much lower than that of the parental and revertant strains. The low level of reactivity may be due to non-specific binding or the presence of another minor phospholipase with homology to Plb1p. In this regard, we reported the cloning of caPLB2, the second PLB gene in C. albicans (Sugiyama et al., 1999).

Our results suggest that Plb1 may directly degrade the phospholipid constituents of host cell membranes. Such injury to the protective cell membrane may provide fungal hyphae with rapid access to the cytoplasm. Similar evidence for enzymic activity by C. albicans in the penetration of glossal epithelium in both rats (Howlett \& Squier, 1980) and humans (Montes \& Wilborn, 1968) has been reported, and studies have been performed that document this process in murine cervical tissue and skin (Scherwitz, 1982). We previously reported a significant difference in the ability of parental and Plb1-deficient strains to penetrate endothelial and epithelial cells in vitro (Leidich et al., 1998). The wildtype parental strain penetrated at twice the rate of the Plb1-deficient strain. However, that study did not include the revertant strain, which was necessary in order to unequivocally ascertain the role of Plb1 in candidal virulence, according to Molecular Koch's Postulates (Falkow, 1988). The successful construction of the revertant and analysis of its virulence in two different animal models enabled us to satisfy Molecular Koch's Postulates and conclusively prove that Plb1p is an important virulence factor in disseminated candidiasis. In the present study, both the parental and the revertant strains penetrated deep into the gastric mucosal and submucosal tissues. In contrast, the Plb1deficient mutant was not as invasive and was generally sequestered to the stomach lumen. The invasiveness of the parental and revertant strains increased their access to the gastric vasculature, thus allowing the organisms to haematogenously disseminate in the bloodstream more efficiently than the Plb1-deficient strain. Consistent with this interpretation, hyphal elements were observed in blood vessel lumens following challenge with the parental or revertant strains, as compared to the mutant. These differences in penetration and dissemination were reflected by the number of candidal c.f.u. recovered from the liver of mice infected with the parental, revertant or Plb1-deficient strains. c.f.u. counts in livers isolated from mice infected with the revertant strain were similar to those obtained from mice infected with the parental strain. In contrast, c.f.u. counts obtained in livers isolated from mice infected with the revertant or parental strain were significantly higher than those found in liver harvested from mice infected with the Plb1-deficient mutant. Similar observations were reported earlier using genetically unrelated strains, which differed in their ability to secrete phospholipase (Barrett-Bee et al., 1985; Ibrahim et al., 1995). The results of our study, using a set of isogenic strains, suggest a possible role for Plb1 in the transmigration of C. albicans across the GI tract, and its ability to cause systemic candidiasis.

Our results show that deleting the PLB1 gene attenuates candidal virulence and reduces the extent of candidal infection in two clinically relevant murine models (intravenous and oral-intragastric models), although this targeted disruption does not render the strains completely avirulent. The fact that this attenuation in virulence is the result of deletion of the PLB1 gene is borne out by our results, which show that when the functional PLB1 gene is reintroduced into the null

\footnotetext{
Fig. 6. Immunofluorescence ( $a, c, e, g)$ and immunoelectron-microscopic, immunogold-labelled images (b, $d, f, h)$ of thin sections of stomachs from C. albicans-infected mice. Sections were examined by immunofluorescence at $20 \mathrm{~d}$ postinfection with the parental strain (a, b), Plb1p-deficient mutant (c, d) and revertant strain (e, f). (g) and (h) Control sections reacted with preimmune serum followed by secondary antibody-FITC or -immunogold label, respectively. CW, Cell wall; pm, plasma membrane. Bars, $50 \mu \mathrm{m}$ (a) and $1.0 \mu \mathrm{m}$ (b).
} 
mutant, the strain (revertant) regains virulence properties. The PLB1 $\Delta^{2}$ strain retains the ability to produce low-grade infections, which is expected because $C$. albicans virulence is believed to be multifactorial. Other putative virulence factors for C. albicans have been the subject of several gene disruption studies. Targeted deletion of genes encoding such factors does not always result in complete avirulence, suggesting that more than one factor may be required for infection. For example, disruption of INT1 supports this concept since int 1 null mutants have a dual phenotype (loss of adherence and germination), both of which have been implicated in candidal virulence (Gale et al., 1998). The int1 null mutants are essentially avirulent $(90 \%$ reduction in mouse mortality). Further investigations regarding the mechanism/s of Plb1 action and how this secreted enzyme relates to other virulence factors will provide important clues about the pathobiology of C. albicans. Uncovering the key components and steps involved in the infectious process of C. albicans should provide an impetus for the development of new antifungal agents and improved therapeutic modalities for efficient treatment of disseminated candidiasis.

\section{ACKNOWLEDGEMENTS}

We thank Dr W. Fonzi for stimulating discussions and invaluable suggestions on the construction of the revertant strain. This work was supported by grant \# AI35097-03 from the National Institutes of Health and grant \# 93-S-507 from the Pfizer Pharmaceutical Group (New York) to M.A.G, and the 2000 Dermatology Foundation - Paul Janssen Research Fellowship to P.K.M.

\section{REFERENCES}

Barrett-Bee, K., Hayes, Y., Wilson, R. G. \& Ryley, J. F. (1985). A comparison of phospholipase activity, cellular adherence and pathogenicity of yeasts. J Gen Microbiol 131, 1217-1221.

Boeke, J. D., LaCroute, F. \& Fink, G. R. (1984). A positive selection for mutants lacking orotidine-5'-phosphate decarboxylase activity in yeast: 5-fluoro-orotic acid resistance. Mol Gen Genet 197, 345-346.

Bruckmann, A., Kunkel, W., Hartl, A., Wetzker, R. \& Eck, R. (2000). A phosphatidylinositol 3-kinase of Candida albicans influences adhesion, filamentous growth and virulence. Microbiology 146, 2755-2764.

Cole, G. T., Lynn, K. T., Seshan, K. R. \& Pope, L. M. (1989). Gastrointestinal and systemic candidosis in immunocompromised mice. J Med Vet Mycol 27, 363-380.

Cole, G. T., Lynn, K. T. \& Seshan, K. R. (1990). An animal model for oropharyngeal, esophageal and gastric candidosis. Mycoses 33, 7-19.

Cole, G. T., Seshan, K. R., Phaneuf, M. \& Lynn, K. T. (1991a). Chlamydospore-like cells of Candida albicans in the gastrointestinal tract of infected, immunocompromised mice. Can J Microbiol 37, 637-646.

Cole, G. T., Kruse, D. \& Seshan, K. R. (1991b). Antigen complex of Coccidioides immitis which elicits a precipitin antibody response in patients. Infect Immun 59, 2434-2446.

Cole, G. T., Halawa, A. A. \& Anaissie, E. J. (1996). The role of the gastrointestinal tract in hematogenous candidiasis: from the laboratory to the bedside. Clin Infect Dis 22 (suppl. 2), S73-S88.
De Backer, M. D., de Hoogt, R. A., Froyen, G., Odds, F. C., Simons, F., Contreras, R. \& Luyten, W. H. (2000). Single allele knock-out of Candida albicans CGT1 leads to unexpected resistance to hygromycin B and elevated temperature. Microbiology 146, 353-365.

De Marie, S. (2000). New developments in the diagnosis and management of invasive fungal infections. Haematologica 85, 88-93.

Edmond, M. B., Wallace, S. E., McClish, D. K., Pfaller, M. A., Jones, R. N. \& Wenzel, R. P. (1999). Nosocomial bloodstream infections in United States hospitals: a three-year analysis. Clin Infect Dis 29, 239-244.

Falkow, S. (1988). Molecular Koch's postulates applied to microbial pathogenicity. Rev Infect Dis 10 (suppl. 2), S274-S276.

Fonzi, W. A. \& Irwin, M. Y. (1993). Isogenic strain construction and gene mapping in Candida albicans. Genetics 134, 717-728.

Gale, C. A., Bendel, C. M., McClellan, M., Hauser, M., Becker, J. M., Berman, J. \& Hostetter, M. K. (1998). Linkage of adhesion, filamentous growth, and virulence in Candida albicans to a single gene, INT1. Science 279, 1355-1358.

Ghannoum, M. A. (2000). Potential role of phospholipases in virulence and fungal pathogenesis. Clin Microbiol Rev 13, 122-143.

Ghannoum, M. A., Spellberg, B., Saporito-Irwin, S. M. \& Fonzi, W. A. (1995). Reduced virulence of Candida albicans PHR1 mutants. Infect Immun 63, 4528-4530.

Gillum, A. M., Tsay, E. Y. \& Kirsch, D. R. (1984). Isolation of the Candida albicans gene for orotidine- 5 -phosphate decarboxylase by complementation of $S$. cerevisiae ura3 and E. coli pyrF mutations. Mol Gen Genet 198, 179-182.

Guentzel, M. N. \& Herrera, C. (1982). Effects of compromising agents on candidosis in mice with persistent infections initiated in infancy. Infect Immun 35, 222-228.

Howlett, J. A. \& Squier, C. A. (1980). Candida albicans ultrastructure: colonization and invasion of oral epithelium. Infect Immun 29, 252-260.

Hube, B., Ruchel, R., Monod, M., Sanglard, D. \& Odds, F. C. (1998). Functional aspects of secreted Candida proteinases. Adv Exp Med Biol 436, 339-344.

Ibrahim, A. S., Mirbod, F., Filler, S. G., Banno, Y., Cole, G. T., Kitajima, Y., Edwards, J. E., Jr, Nozawa, Y. \& Ghannoum, M. A. (1995). Evidence implicating phospholipase as a virulence factor of Candida albicans. Infect Immun 63, 1993-1998.

Jarvis, W. R. (1995). Epidemiology of nosocomial fungal infections, with emphasis on Candida species. Clin Infect Dis 20, 1526-1530.

Klepser, M. E., Lewis, R. E. \& Pfaller, M. A. (1998). Therapy of Candida infections: susceptibility testing, resistance, and therapeutic options. Ann Pharmacother 32, 1353-1361.

Klotz, S. A., Drutz, D. J., Harrison, J. L. \& Huppert, M. (1983). Adherence and penetration of vascular endothelium by Candida yeasts. Infect Immun 42, 374-384.

Kruse, D. \& Cole, G. T. (1992). A seroreactive 120 -kilodalton $\beta-1,3-$ glucanase of Coccidioides immitis which may participate in spherule morphogenesis. Infect Immun 60, 4350-4363.

Leidich, S. D., Ibrahim, A. S., Fu, Y. \& 8 other authors (1998). Cloning and disruption of caPLB1, a phospholipase B gene involved in the pathogenicity of Candida albicans. J Biol Chem 273, 26078-26086.

Luna, L. G. (1968). Manual of Histological Staining Methods of the Armed Forces Institute of Pathology, 3rd edn. New York: McGraw-Hill. 
Montes, L. F. \& Wilborn, W. H. (1968). Ultrastructural features of host-parasite relationship in oral candidiasis. J Bacteriol 96, 1349-1356.

Mukherjee, P. K. \& Ghannoum, M. A. (2001). Secretory proteins in fungal virulence. In Fungal Pathogenesis: Principles and Clinical Applications, pp. 51-79. Edited by R. A. Calderone \& R. L. Cihlar. New York: Marcel Dekker.

Odds, F. C. (1994). Candida albicans, the life and times of a pathogenic yeast. J Med Vet Mycol 32 (suppl. 1), 1-8.

Odom, R. B. (1994). Common superficial fungal infections in immunocompromised patients. J Am Acad Dermatol 31, S56-S59.

Pagano, L., Antinori, A., Ammassari, A. \& 11 other authors (1999). Retrospective study of candidemia in patients with hematological malignancies. Clinical features, risk factors and outcome of 76 episodes. Eur J Haematol 63, 77-85.

Patterson, T. \& Andriole, V. T. (1989). The role of liposomal amphotericin B in the treatment of systemic fungal infections. Eur J Clin Microbiol 25, S63.

Pope, L. M., Cole, G. T., Guentzel, M. N. \& Berry, L. J. (1979). Systemic and gastrointestinal candidiasis of infant mice after intragastric challenge. Infect Immun 25, 702-707.

Pugh, D. \& Cawson, R. A. (1977). The cytochemical localization of phospholipase in Candida albicans infecting the chick chorioallantoic membrane. Sabouraudia 15, 29-35.

Ravdin, J. I., Murphy, C. F., Guerrant, R. L. \& Long-Krug, S. A. (1985). Effect of antagonists of calcium and phospholipase A on the cytopathogenicity of Entamoeba histolytica. J Infect Dis 152, 542-549.

Rose, M. D., Winston, F. \& Hieter, P. (1990). Methods in Yeast Genetics: a Laboratory Course Manual. Cold Spring Harbor, NY: Cold Spring Harbor Laboratory.

Saffer, L. D. \& Schwartzman, J. D. (1991). A soluble phospholipase of Toxoplasma gondii associated with host cell penetration. $J$ Protozool 38, 454-460.

Sambrook, J., Fritsch, E. F. \& Maniatis, T. (1989). Molecular Cloning: a Laboratory Manual, 2nd edn. Cold Spring Harbor, NY: Cold Spring Harbor Laboratory.

Scherwitz, C. (1982). Ultrastructure of human cutaneous candidosis. J Investig Dermatol 78, 200-205.
Schmiel, D. H. \& Miller, V. L. (1999). Bacterial phospholipases and pathogenesis. Microbes Infect 1, 1103-1112.

Seshan, K. R. \& Cole, G. T. (1994). Structural studies of Coccidioides immitis. In Molecular Biology of Pathogenic Fungi, pp. 265-273. Edited by B. Maresca \& G. S. Kobayashi. New York: Telos Press.

Silverman, D. J., Santucci, L. A., Meyers, N. \& Sekeyova, Z. (1992). Penetration of hosts by Rickettsia rickettsii appears to be mediated by a phospholipase of rickettsial origin. Infect Immun 60, 2733-2740.

Spurr, A. R. (1969). A low-viscosity epoxy resin embedding medium for electron microscopy. J Ultrastruct Res 226, 31-43.

Sugiyama, Y., Nakashima, S., Mirbod, F., Kanoh, H., Kitajima, Y., Ghannoum, M. A. \& Nozawa, Y. (1999). Molecular cloning of a second phospholipase B gene, caPLB2 from Candida albicans. Med Mycol 37, 61-67.

Viola, A. M., Lodi, T. \& Ferrero, I. (1999). A Klaac null mutant of Kluyveromyces lactis is complemented by a single copy of the Saccharomyces cerevisiae AAC1 gene. Curr Genet 36, 29-36.

Viscoli, C., Girmenia, C., Marinus, A. \& 9 other authors (1999). Candidemia in cancer patients: a prospective, multicenter surveillance study by the Invasive Fungal Infection Group (IFIG) of the European Organization for Research and Treatment of Cancer (EORTC). Clin Infect Dis 28, 1071-1079.

Wey, S. B., Mori, M., Pfaller, M. A., Woolson, R. F. \& Wenzel, R. P. (1988). Hospital-acquired candidemia. The attributable mortality and excess length of stay. Arch Intern Med 148, 2642-2645.

Wey, S. B., Mori, M., Pfaller, M. A., Woolson, R. F. \& Wenzel, R. P. (1989). Risk factors for hospital-acquired candidemia. A matched case-control study. Arch Intern Med 149, 2349-2353.

Zhou, D., Chen, L. M., Hernandez, L., Shears, S. B. \& Galan, J. E. (2001). A Salmonella inositol polyphosphatase acts in conjunction with other bacterial effectors to promote host cell actin cytoskeleton rearrangements and bacterial internalization. Mol Microbiol 39, 248-259.

Received 29 January 2001; revised 23 April 2001; accepted 11 May 2001. 